Des actions en faveur de la nature dans les cours d'école : un levier pertinent pour mieux vivre ensemble dans une démarche d'établissement "vers un développement durable " Classroom actions that favour nature: learning to share the planet through a school project for sustainable development Algunas acciones en favor de la naturaleza en clase: un dispositivo apropiado para mejor vivir juntos el impulso del desarrollo sustentable de un establecimiento escolar

\title{
Véronique Philippot
}

Volume 37, numéro 2, automne 2009

Vivre ensemble, sur Terre

URI : https://id.erudit.org/iderudit/038817ar

DOI : https://doi.org/10.7202/038817ar

\section{Aller au sommaire du numéro}

\section{Éditeur(s)}

Association canadienne d'éducation de langue française

\section{ISSN}

0849-1089 (imprimé)

1916-8659 (numérique)

Découvrir la revue

\section{Citer cet article}

Philippot, V. (2009). Des actions en faveur de la nature dans les cours d'école : un levier pertinent pour mieux vivre ensemble dans une démarche d'établissement « vers un développement durable ». Éducation et francophonie, 37(2), 79-97. https://doi.org/10.7202/038817ar

\section{Résumé de l'article}

Dans le cadre des pratiques développées par les classes-ateliers-environnement de Tours (France) qui accompagnent des projets d'éducation à l'environnement avec les écoles primaires, celles qui visent à réintroduire un peu de nature dans les cours des écoles font ici l'objet d'une première analyse. Une exploration bibliographique sur le sujet permet d'argumenter tout l'intérêt de ce type d'actions concrètes, intérêt déjà perçu intuitivement par la communauté éducative sollicitée. Pourtant, une analyse préliminaire de ces actions pédagogiques met en évidence des difficultés à différents niveaux qu'il serait intéressant d'étudier de manière approfondie par la suite. Cet article tend également à montrer qu'une volonté de renaturalisation de l'école pourrait s'avérer une entrée pertinente pour des établissements qui s'engagent dans une démarche de développement durable. En effet, le bien-fondé des actions collectives visant à restaurer un peu de biodiversité dans un espace éducatif informel peut s'ancrer dans un champ philosophique qui explore le thème du mieux-vivre ensemble à l'école.
Tous droits réservés (C) Association canadienne d'éducation de langue française, 2009
Cest protégé par la loi sur le droit d'auteur. L'utilisation des services d'Érudit (y compris la reproduction) est assujettie à sa politique d'utilisation que vous pouvez consulter en ligne. 


\section{Des actions en faveur de la nature dans les cours d'école : un levier pertinent pour mieux vivre ensemble dans une démarche d'établissement " vers un développement durable »}

\section{Véronique PHILIPPOT}

Collège André-Bauchant, Château-Renault, France

\section{RÉSUMÉ}

Dans le cadre des pratiques développées par les classes-ateliers-environnement de Tours (France) qui accompagnent des projets d'éducation à l'environnement avec les écoles primaires, celles qui visent à réintroduire un peu de nature dans les cours des écoles font ici l'objet d'une première analyse. Une exploration bibliographique sur le sujet permet d'argumenter tout l'intérêt de ce type d'actions concrètes, intérêt déjà perçu intuitivement par la communauté éducative sollicitée. Pourtant, une analyse préliminaire de ces actions pédagogiques met en évidence des difficultés à différents niveaux qu'il serait intéressant d'étudier de manière approfondie par la suite. Cet article tend également à montrer qu'une volonté de renaturalisation de l'école pourrait s'avérer une entrée pertinente pour des établissements qui s'engagent dans une démarche de développement durable. En effet, le bien-fondé 
Des actions en faveur de la nature dans les cours d'école: un levier pertinent pour mieux vivre ensemble

dans une démarche d'établissement «vers un développement durable»

des actions collectives visant à restaurer un peu de biodiversité dans un espace éducatif informel peut s'ancrer dans un champ philosophique qui explore le thème du mieux-vivre ensemble à l'école.

\section{ABSTRACT}

\section{Classroom actions that favour nature: learning to share the planet through a school project for sustainable development}

Véronique PHILIPPOT

André-Bauchant College, Château-Renault, France

This article analyzes practices developed by environment workshop-classes in Tours (France) that complement environmental education projects in elementary schools. These practices aim to bring a little nature back into the classroom. A bibliographical exploration of the subject shows increased interest in this type of action. The educational community that was approached had already perceived this interest intuitively. However, a preliminary analysis of these pedagogical actions reveals problems at different levels, which would be interesting to study in more depth. The article also attempts to show that the school's desire to renaturalize could be a relevant port of entry for schools involved in a sustainable development process. The legitimacy of collective actions aimed at restoring a little biodiversity to an informal educational space can be based on a philosophical exploration based on the theme of sharing the planet.

\section{RESUMEN}

\section{Algunas acciones en favor de la naturaleza en clase: un dispositivo apropiado para mejor vivir juntos el impulso del desarrollo sustentable de un establecimiento escolar.}

Véronique PHILIPPOT

Colegio André-Bauchant, Château-Renault, Francia

En el cuadro de acciones desarrolladas por las clases-talleres-medio-ambiente de Tours (Francia), que acompañan los proyectos de educación relativas al entorno ecológico con escuelas primarias, aquellas que apuntaban la reintroducción de un poco de naturaleza en los cursos constituyen aquí los objetos de un primer análisis. Una exploración bibliográfica sobre el tema permite argumentar el interés de ese tipo de acciones concretas, interés ya percibido intuitivamente por la comunidad educativa solicitada. Sin embargo, el análisis preliminar de dichas acciones pedagógicas pone en evidencia dificultades en diferentes niveles, que sería interesante 
Des actions en faveur de la nature dans les cours d'école: un levier pertinent pour mieux vivre ensemble

dans une démarche d'établissement «vers un développement durable»

profundizar posteriormente. Este artículo tiende a mostrar que una voluntad de re-naturalización de la escuela podría ser una entrada apropiada para las escuelas que adhieren al proyecto de desarrollo sustentable. En efecto, lo bien fundado de las acciones colectivas que tratan restaurar un poco de biodiversidad en un espacio educativo informal pueden cimentarse en un campo filosófico que explora el tema de mejorar el vivir juntos en la escuela.

\section{Introduction}

Si l'on m'interroge à propos de ma sensibilité aux paysages des cours d'école, je voyage volontiers dans mon propre répertoire de souvenirs d'élève. Ce processus que Dominique Bachelart (2009) qualifie d'exploration autobiographique permet de s'engager «dans un processus actif d'interprétation et de construction de signification qui laisse une empreinte cognitive».

$\mathrm{Si}$ je faisais ce récit de nature réflexive (relative à la narration de soi), mes cours d'école seraient grises et froides. Nos jeux furent un temps de gratter un coin de terre battue oublié au pied des platanes pour obtenir du sable fin. De hauts murs salis de lambeaux de vie verdâtres plongeaient dans l'ombre l'espace dédié aux récréations. La porte en chêne toujours fermée sur nos rêves d'espace refrénait mon humeur entreprenante. Beaucoup plus tard, cette sensation d'enfermement m'a hantée et, devenue enseignante, j'ai désiré ouvrir l'école à la nature, à la lumière, au monde.

Aujourd'hui, ma posture a évolué vers celle d'une professeure spécialisée en l'éducation à l'environnement auprès d'enfants du primaire et je me suis intéressée à la question des cours d'école. Les contacts que j'exerçais, enfant, avec la nature en dehors de l'école étaient intenses et répétés; l'impact de l'environnement scolaire fut ainsi négligeable. Mais qu'en est-il aujourd'hui de la jeune population urbaine et de ses rapports avec la cour de récréation? L'équipe de Diane Pruneau fait état des conséquences fâcheuses d'un environnement urbain développé autour de l'automobile sur la santé physique et morale des enfants (Pruneau et Desjardins, 2003). La cour serait en effet une continuité du paysage du quartier, empruntant ses matériaux artificiels et son mobilier urbain.

Cet article dresse un premier bilan d'expériences menées dans le cadre d'un dispositif éducatif proposé aux écoles de Tours. Elles rejoignent d'autres démarches déjà diffusées et discutées par d'autres praticiens et théoriciens en éducation à l'environnement, et qui concernent l'introduction d'éléments de nature dans les cours à une échelle plus globale. Après un survol succinct de travaux interrogeant les liens entre le jeune et la nature, une exploration de la cour est proposée en s'exerçant à un angle de vision écocentrique, la cour étant ici considérée comme un tout, une entité écologique englobant l'humain et digne de considération morale. Puis, à la lumière de supports bibliographiques, on s'interrogera sur la vocation fonctionnelle 
Des actions en faveur de la nature dans les cours d'école: un levier pertinent pour mieux vivre ensemble

dans une démarche d'établissement «vers un développement durable»

et informelle des lieux de récréation où nos jeunes apprenants passent une grande partie de leur temps. Enfin, nourrie par le vécu des pratiques développées à Tours, je défendrai l'idée que les procédures d'établissement identifiées en démarche de développement durable $e^{1}$ ne pourraient faire abstraction d'une éducation à la nature, ni même sous-estimer cette dimension écologique dans une recherche de valeurs d'altruisme contribuant au bien-vivre communautaire.

\section{Brève exploration bibliographique montrant l'importance de l'éveil à la nature pendant la jeunesse}

Beaucoup d'études existent sur le sujet et quelques éléments de réflexion sont abordés ici. Des travaux explorant les sources d'équilibre et de bien-être chez les adultes montrent l'impact positif de la nature pendant l'enfance. Ainsi, selon la pionnière Edith Cobb dans ce domaine de recherche, la santé mentale dynamique des adultes serait étroitement liée à la créativité. Le rapport de James Raffan (2002) interprète ainsi une conclusion d'Edith Cobb tirée de son ouvrage précurseur de 1977 : «L'expression créative est enracinée dans la relation de l'enfant avec la complexité, la plasticité et la maniabilité du monde naturel.» La nature fournirait donc un champ expérientiel d'investigation infini et propice à l'épanouissement du pouvoir créateur, et, selon Edith Cobb, clé d'un sentiment durable de quiétude apaisante.

Plus récemment, les recherches exploratrices dirigées par Diane Pruneau (2002) chez des adolescents montrent que «la relation enfantine de contact symbiotique et étroit avec le milieu naturel (décrite par Edith Cobb) était au départ peu présente chez les adolescents interrogés ». Cependant, « elle peut être réanimée par des activités d'une demi-journée de contact sensoriel solitaire ou d'action environnementale ", ce qui leur permet de "découvrir ou redécouvrir un sens et une importance au milieu naturel ». Toujours selon l'auteure, une action en faveur de la nature (par un processus volontaire, lucide et responsable) leur apporte « une grande satisfaction et un sentiment de pouvoir " qui répond fort bien au besoin de défi vis-à-vis de soi et d'autrui propre à ce stade de développement. En outre, vivre ensemble des activités dans et pour la nature répond aussi à leurs besoins de s'émerveiller, de rêver, de vivre des aventures et de rencontrer l'autre (humain ou pas). L'enquête de Diane Pruneau (2002) auprès des jeunes met enfin en évidence le fait que la nature leur apporte le calme et la sérénité dont ils s'estiment en manque.

De façon plus pragmatique, des études effectuées à l'échelle des États-Unis (Lieberman et Hoody, 1998) montrent que les rendements scolaires seraient améliorés lorsque la nature est utilisée comme cadre d'apprentissage. En outre, les enseignants

1. Je fais référence ici à une perspective d'établissement "en démarche de développement durable », puisqu'il s'agit de la porte d'entrée institutionnelle actuellement en France. Je considère aussi que l'acte d'éduquer est un acte politique qui concerne les enjeux communautaires. Dans ce cadre, l'éducation à l'environnement a une place prépondérante pour la construction d'une communauté pour laquelle les efforts sont concentrés vers le développement de compétences esthétiques afin d'appréhender les beautés de la nature et les compétences critiques. La connaissance du monde (ici et ailleurs) et le sentiment d'appartenance au monde sont essentiels pour nourrir cette aptitude à la critique. 
notent une augmentation des capacités d'engagement et d'enthousiasme des élèves, tandis que beaucoup mentionnent aussi une meilleure adaptabilité aux styles d'enseignement et un effort de courtoisie envers les autres.

Ainsi, la cour d'école étant le périmètre extérieur immédiat susceptible d'offrir des îlots de nature aux écoliers, la présente étude se focalise sur cet espace collectif disponible qu'il convient au préalable de considérer sous un angle descriptif critique.

\section{La cour d'école, un milieu de vie teinté d'anthropocentrisme}

\section{La cour, un espace de socialisation avant tout}

Les écoles jouissent normalement d'un espace réservé, soigneusement clos, parfois ceint de murs infranchissables. Le rapport à la cour est certes fonctionnel, mais ce lieu revêt également une dimension affective chez les plus jeunes et les paysages de cours occupent une grande place dans la collection mentale des clichés d'enfance. Dans cet espace partagé se tissent aussi les premiers liens avec autrui hors de la sphère familiale, l'espace de récréation étant un lieu social gouverné par des règles collectives imposées ou implicites. Le sable de la cour, les feuilles mortes et les petits cailloux font l'objet de maintes expériences partagées sensorielles ou cognitives et contribuent au développement individuel. Dans cet espace investi et exploré dans les moindres recoins, l'enfant doit affirmer sa territorialité et revendiquer son espace de jeux ou de repos.

\section{La cour, un milieu de vie réservé aux humains}

Si l'on considère la cour comme milieu physique et avec le regard d'un écologue, ce lieu est une représentation quasi caricaturale de l'humanité qui tend à accaparer l'espace sans souci des répercussions silencieuses sur l'ordre naturel. En cela, l'école reproduit en petit l'état de notre écosphère où l'évolution normale des communautés biologiques est compromise, gouvernée selon un mode anthropocentrique, appauvrie du sauvage ancestral. Le terrain artificialisé à vocation récréative est l'un des théâtres de la désertification biologique dont l'humain est responsable. Cela traduirait-il l'inquiétude suscitée par le vivant non domestiqué? L'espace de jeux qui devrait être propice à de multiples expériences est dénaturé, imperméabilisé, nivelé, uniformisé... Un monde minéral figé et inerte a remplacé la couverture végétale primitive. Le processus vital est ici presque suspendu.

L'expression du vivant est en effet déterminée par quatre éléments universels à connotation philosophique : le feu (le soleil), l'air, la terre (le sol) et l'eau. Deux paramètres vitaux sont généralement malmenés dans la cour: le sol et l'eau. D'abord, tout se passe comme si, en contradiction avec les fondamentaux que tout élève engrange, on avait oublié que la matière qui constitue le sol est le creuset fertile de toute vie, la mère nourricière de l'humanité. Elle incarne souvent saleté et vermine dans les représentations premières des enfants et des enseignants. Ainsi les espaces autour des écoles ont-ils été enrobés en prétextant le danger des cailloux saillants ou 
la boue collée aux petits pieds. La cour est devenue le prolongement de la salle de classe. Ensuite, l'eau de l'école jouit d'une connotation plutôt positive pourvue qu'elle coule du robinet, limpide et aseptisée. Les gouttes de pluie touchent à peine le sol imperméable qu'elles ruissellent vers les bouches d'un réseau souterrain, source d'un imaginaire fécond chez les enfants. Que devient l'eau de la lumière dans ce monde des ténèbres? L'Homme aurait-il là aussi contourné les lois naturelles qui veulent que la pluie mouille le sol et soit absorbée par les racines des plantes? L'observation directe (moment informel qui échappe aux stratégies pédagogiques maîtrisées par l'adulte) des phénomènes physiques de la cour ne nous enseigne pas le cycle normal de l'eau.

Aussi comment, dans ces conditions, la cour peut-elle être appréhendée comme un milieu de vie ordinaire? Familier mais pas ordinaire. La vie qui s'y manifeste cependant, petits îlots écologiques dans un océan figé d'asphalte, relève de conditions extrêmes : amplitudes de température significatives, pénurie de sol, sécheresse, insolations, vents que rien ne freine à terre, apports massifs d'eau lors des pluies d'orage... Ainsi, profitant parfois de vieux murs aux pierres disjointes, un cortège de plantes dites pionnières s'accrochent à la vie. En outre, de fortes contraintes s'exercent à l'encontre des communautés vivantes par la présence intermittente d'une population d'enfants très dense.

En somme, deux impératifs tyrannisent la gestion de cet espace: sécurité et hygiène. La nature devient ici potentiellement dangereuse et sale. Ainsi, les branches mortes sont élaguées avant leur chute et les feuilles tombées sont autant de risques de glissades. Les baies tolérées dans les espaces dédiés aux enfants sont rares en raison du risque d'empoisonnement. L'assainissement des cours conduit à leur artificialisation souvent très poussée. Cet état de fait ne pourrait-il pas rendre perplexes les élèves, au regard des schémas théoriques des cycles de la matière péniblement construits pendant la leçon de sciences? L'investigation bibliographique qui suit permet d'élargir ce point de vue intuitif.

\section{Pourquoi prendre en considération la cour d'école dans les stratégies d'enseignement?}

Cette section s'inspire de l'analyse documentaire d'une équipe de recherche pluridisciplinaire dirigée par James Raffan (2002). Elle rassemble et met en interférence les recherches d'experts dans diverses disciplines, de l'architecte-paysagiste au praticien. Elle a suscité une analyse de mes propres pratiques et permet d'ancrer celles-ci dans une problématique plus globale.

Ainsi, d'après l'étude de James Raffan, l'idée de recentrer les actions relatives à une éducation à la biodiversité dans la cour d'école n'est pas une idée émergente ni orpheline. Les défenseurs de projets visant à reverdir cet espace initialement destiné à la surveillance passive et aux jeux participent au mouvement historique de l'Éducation nouvelle lancé dans les années 1930. Les pères philosophiques de tels bouleversements idéologiques, dont Rousseau (1712-1852), prônent une éducation 
davantage centrée sur le développement biologique de l'enfant. Le champ expérientiel prendrait alors une importance fondamentale. Les éléments biophysiques qui structurent le paysage immédiat des lieux d'éducation interféreraient insidieusement dans les procédures cognitives conscientes et formelles. Dominique Bachelart (2009) traite de la "force de la sphère informelle» sur les jeunes apprenants. Au même titre que les domaines intimes et familiaux, la cour d'école serait «placée très bas dans la pyramide des lieux d'éducation comme porteuse d'un savoir incohérent, non crucial, moins digne d'intérêt». Et pourtant, d'après l'auteure, «c'est dans la sphère informelle que s'acquièrent la plupart des apprentissages jugés significatifs ».

D'après James Raffan, qui dresse un aperçu du contexte historique des actions de revitalisation engagées dans des établissements québécois, «la naturalisation des cours d'école est sans doute l'un des échos contemporains les plus persistants de l'Éducation nouvelle». L'auteur du rapport précité s'appuie notamment sur les travaux de la Britannique Eileen Adams (1990) qui déclare que «l'école et sa cour doivent constituer un environnement complet [...] bon nombre de cours sont, au pire, des espaces stériles et venteux dont l'herbe est coupée à ras ou des étendues d'asphalte qui contribuent très peu au programme éducationnel ou à l'environnement en général». Les constats sévères de l'enquête outre-Manche s'articulent autour de l'idée que l'état des cours nuit en général aux enseignements dispensés en classe en diffusant des messages négatifs, imprégnés de morosité, de violence et de contraintes. Ces extérieurs s'identifient plus à des cours de prison qu'à des lieux d'incitation à la paix et la rêverie. Ils seraient assimilables à des lieux d'éradication du sauvage.

Or, l'enquête de l'équipe d'Eileen Adam (1990) conclut que «l'aspect de l'école reflète la philosophie de l'établissement et communique subtilement un peu de son attitude aux jeunes et les informe de la valeur que nous leur accordons». L'une des éthiques fondamentales en matière d'éducation à l'environnement étant le respect du vivant et une certaine forme de compassion à l'égard de toute entité vitale, on peut s'interroger sur les contradictions entre le discours et les pratiques d'établissement. La cour d'école renvoie des messages négatifs et fondés sur un anthropocentrisme exacerbé au regard de la gestion peu subtile des mauvaises herbes, des butineurs à dard, des petites bêtes égarées dans le désert de bitume ou même des feuilles mortes brusquement indésirables dès lors qu'elles touchent le substrat hostile au pied des marronniers. Peut-on recevoir l'enseignement du cycle du carbone tout en observant les feuilles des arbres cramoisies bruyamment soufflées comme de vulgaires déchets et disparaissant dans les conteneurs-poubelles? Notre remise en question devrait commencer là : peut-on concevoir un projet naturaliste si l'on ne revalorise pas ces extraordinaires petits panneaux solaires teintés de rouille, qui ont bien servi la communauté végétale? Ces entités vivantes appartiennent au cycle de la matière et doivent retourner en poussière pour que l'arbre perdure. C'est l'exemple premier du développement durable, dans le sens d'une pérennisation d'un processus évolutif normal, celui de l'arbre qui renaît de lui-même, de sa propre matière décomposée à ses pieds.

Wendy Titman (1994) n’hésite pas à évoquer « le programme éducationnel caché dans la cour d'école». Considérant le temps passé par les enfants dans ces îlots 
écologiquement désertiques, ce programme informel « devient une force nuisible » et d'autres auteurs écrivent que «les cours d'école ont le potentiel de compromettre l'apprentissage » (Cheskey et Pearce, 1994). Inversement, le rapport de James Raffan fait état des recherches de Robin Moore (1989) qui poursuit les travaux préliminaires d'Edith Cobb. Il affirme que les cours offrant une diversité de choix aux enfants inspirent des jeux plus créatifs, ce qui améliore les interactions sociales et les facultés d'expression. Le développement intellectuel serait globalement favorisé. Robin Moore souligne le droit à l'enfant à un environnement diversifié et prétend que la meilleure façon d'y parvenir est la naturalisation.

\section{Des projets pour accueillir un peu de nature dans les cours d'école à Tours (France) : contexte et analyse des constats}

\section{Un dispositif d'éducation à l'environnement à Tours}

Les classes-ateliers-environnement de Tours sont nées en 2002 d'une volonté partagée entre l'élue chargée des affaires scolaires et une inspectrice de l'Éducation nationale. Ce dispositif s'adresse aux quelque 35 écoles élémentaires de la ville et la participation des 18 classes par an repose sur le volontariat. Les projets se déroulent en classe transplantée pendant un trimestre à raison d'une journée par semaine. Cependant, les enseignants peuvent préparer ou prolonger les activités liées au projet sur le restant de l'année scolaire en bénéficiant de l'accompagnement du dispositif.

La méthodologie relève d'une pédagogie de projet à travers laquelle la situation initiale est soigneusement clarifiée et les axes de progrès définis avec les enfants. Afin de tendre vers le changement attendu, des situations d'apprentissages pluridisciplinaires en lien avec les programmes scolaires sont mises en place et des structures partenaires peuvent être sollicitées ponctuellement. Le projet de classe est vécu comme une aventure collective concrète où le mieux-vivre ensemble entre humains ou non-humains est un défi permanent.

Lorsque les classes-ateliers-environnement furent pensées par l'élue et l'inspectrice, l'idée de mener des actions dans les écoles elles-mêmes ne fut pas évoquée, alors qu'un autre projet municipal baptisé La cour merveilleuse était en marche. Lors des négociations préliminaires, j'ai présenté ce que je croyais bon à la fois pour les enfants et pour l'environnement: oser des coins de nature dans la cour. J'avais naïvement associé le thème du merveilleux avec un acteur que je m'imaginais incontournable, la nature. J'ai depuis mesuré l'ampleur des difficultés que cette idée d'abord perçue comme fantaisiste a suscitées.

Sur 104 classes engagées dans des projets relatifs à l'environnement et au développement durable, 65 projets se sont développés autour d'un thème à forte connotation naturaliste parmi lesquels 19 ont donné lieu à des réalisations menées dans la cour de l'école. Certains cependant ont été ajournés ou même abandonnés. Le tableau 1 fait état des actions programmées et montre que seulement 11 projets ont reverdi le paysage scolaire. 
Des actions en faveur de la nature dans les cours d'école: un levier pertinent pour mieux vivre ensemble dans une démarche d'établissement «vers un développement durable»

Tableau 1. Inventaire des projets aboutis ou non ayant donné lieu à des actions concrètes en faveur de la nature dans les cours d'école

\begin{tabular}{|c|c|c|c|c|c|}
\hline & $\begin{array}{l}\text { Année } \\
\text { scolaire }\end{array}$ & $\begin{array}{l}\text { Nombre } \\
\text { de classes } \\
\text { concernées }\end{array}$ & $\begin{array}{l}\text { Niveau de classe } \\
\text { et âge moyen }\end{array}$ & Nom de l'école & $\begin{array}{l}\text { Nature du projet } \\
\text { dans l'école }\end{array}$ \\
\hline 1 & $2002-2003$ & 3 & CE2+CM2 (8-11 ans) & RACAULT & Plantation d'une haie \\
\hline 2 & $2002-2003$ & 2 & CM (9-10 ans) & ALAIN & $\begin{array}{l}\text { Amélioration du bassin } \\
\text { existant pour créer une petite } \\
\text { réserve naturelle }\end{array}$ \\
\hline 3 & $2002-2003$ & 1 & CP (6-7 ans) & MACÉ & Jardin \\
\hline 4 & $2002-2003$ & 2 & CE2 (8-9 ans) & BERNARD & Plantation d'une haie \\
\hline 5 & $2003-2004$ & 2 & CM2 (10-11 ans) & PÉGUY & $\begin{array}{l}\text { Gestion de la surface } \\
\text { engazonnée en prairie pour } \\
\text { protéger les orchidées }\end{array}$ \\
\hline 6 & $2004-2005$ & 2 & CLIS + CE1 (6-8 ans) & DIDEROT & Plantation d'une haie \\
\hline 7 & $2004-2005$ & 2 & CM1 + CM2 (9-11 ans) & PÉGUY & $\begin{array}{l}\text { Création d'une petite mare } \\
\text { à l'école }\end{array}$ \\
\hline 8 & $2004-2005$ & 1 & CE2 (8-9 ans) & SAINT-EXUPÉRY & Jardinage \\
\hline 9 & $2004-2005$ & 2 & CM1 + CM2 (9-11 ans) & PAUL-BERT & $\begin{array}{c}\text { Protection des migrateurs } \\
\text { dans I'école, aménagement } \\
\text { du grenier }\end{array}$ \\
\hline 10 & $2004-2005$ & 1 & CE1 (7-8 ans) & CHATEAUBRIAND & $\begin{array}{c}\text { Zone de protection des } \\
\text { orchidées }\end{array}$ \\
\hline 11 & $2004-2005$ & 1 & CP (6-7 ans) & MIRABEAU & $\begin{array}{l}\text { Mini-réserve pour petits } \\
\text { animaux }\end{array}$ \\
\hline 12 & $2005-2006$ & 1 & CM2 & RACAULT & Plantation d'une haie \\
\hline 13 & $2006-2007$ & 2 & $\mathrm{CM} 2+\mathrm{CE} 1$ & RASPAIL & Plantation d'une haie \\
\hline 14 & $2006-2007$ & 1 & $\mathrm{CE} 1+\mathrm{CE} 2$ & ANATOLE FRANCE & Jardin \\
\hline 15 & 2006-2007 & 2 & CE2 & GIDE & Pose de nichoirs \\
\hline 16 & $2007-2008$ & 1 & $\mathrm{CP}$ & BASTIÉ & $\begin{array}{l}\text { Création d'une mini-zone } \\
\text { humide }\end{array}$ \\
\hline 17 & $2007-2008$ & 1 & CE1 & ROMAIN-ROLLAND & $\begin{array}{l}\text { Construction d'un muret } \\
\text { de pierres }\end{array}$ \\
\hline 18 & $2007-2008$ & 1 & $\mathrm{CE} 2+\mathrm{CM} 1+\mathrm{CM} 2$ & PÉROCHON & Jardin pour les papillons \\
\hline 19 & $2007-2008$ & 1 & $\mathrm{CP}$ & RASPAIL & $\begin{array}{l}\text { Plantation d'arbres à } \\
\text { papillons pour les butineurs }\end{array}$ \\
\hline
\end{tabular}

Projets concrétisés dans la période prévue

Projets ajournés et concrétisés ultérieurement

Projets non aboutis 


\section{Analyse préliminaire des difficultés rencontrées}

Étant avant tout enseignante et praticienne en éducation à l'environnement, je n'avais pas élaboré en amont des projets de protocoles précis en vue de collectes de données destinées aux futures analyses. Cette étude trouve donc ses limites dans son caractère pragmatique et fait d'abord état de constats qui invitent certes à des réflexions plus poussées.

Aussi, l'identification des paramètres ayant pu entraver la bonne évolution de projets concernant l'enceinte scolaire n'est pas toujours aisée et leur influence relative est encore moins facilement évaluable. Cependant, cette étude préalable montre qu'au moins dix aspects contribueraient à expliquer les obstacles rencontrés. Ils sont répertoriés par ordre décroissant d'influence estimée sur la globalité des 19 projets :

1. Les contraintes relevant de la législation française en matière d'hygiène et de sécurité.

2. Le fait que le projet ne soit pas inscrit dans le projet d'école et qu'il n'ait pas été soumis à l'approbation de l'équipe pédagogique de façon formelle.

3. Le cloisonnement entre les services municipaux qui rend la coordination des interventions difficile ou mal comprise.

4. L'instabilité des personnes en poste, qui peut compromettre la pérennisation de projets reposant essentiellement sur des motivations individuelles.

5. Les difficultés techniques mal appréhendées en amont.

6. Une démotivation des enseignants en cours de projet du fait d'une implication initiale insuffisante et d'un manque de conscience de l'intérêt d'une éducation à l'environnement.

7. Le manque de concertation préalable entre le personnel non enseignant et les enseignants.

8. La crainte des réactions des parents ou des résidents du quartier.

9. Le coût financier mal pris en considération ou mal évalué au départ.

10. Le manque de réactivité et d'implication du partenariat sollicité.

Cet inventaire est né de mes propres observations et d'analyses qui n'ont pu faire à ce jour l'objet d'échanges formalisés et encore moins validés par les équipes éducatives ayant participé aux projets de naturalisation. Ce travail préliminaire demanderait donc à être corroboré par une enquête auprès des acteurs adultes en utilisant des protocoles appropriés. En outre, ayant eu moi-même un rôle opérationnel important lors de la négociation et du déroulement des projets, il serait opportun de prendre en considération l'implication d'un acteur dont la conscience écologique affichée aurait pu agir comme un frein. En effet, la vision extérieure et neuve que j'ai apportée sur leur lieu communautaire, associée à une envie d'agir enthousiaste, a pu déconcerter des enseignants auxquels la situation paraissait échapper. Les choses ainsi posées, quelques enseignements peuvent déjà émaner de ces expérimentations concrètes dans les écoles. 


\section{Une réglementation peu encourageante}

La première source d'entrave tient à une législation sévère dont la finalité est de réduire au maximum les expositions aux dangers. La prise de connaissance des règles inhérentes aux lieux fréquentés par des enfants, parfois peu aisées à obtenir et interpréter, rebute souvent les enseignants. À travers nos projets, il nous a fallu notamment prendre en considération la réglementation fixant la liste des plantes interdites dans les cours d'école (toxiques ou pouvant blesser) et celle concernant les points d'eau de surface accessibles aux enfants. Cette dernière réduit considérablement le champ des actions autorisées et son application stricte a eu pour effet de briser des projets de classe déjà bien avancés, même lorsque les négociations avec les acteurs avaient déjà été engagées.

\section{Une nécessaire concertation et appropriation des projets par les différents acteurs}

Ensuite, la nature des facteurs limitant ou gênant la concrétisation de projets d'aménagement dans les cours dépend de la nature des projets eux-mêmes. Lorsque les décisions concernent un changement de la topographie des lieux (création d'une zone revitalisée par enlèvement de l'asphalte, affouillement du substrat, construction pérenne comme un mur de pierre...), divers services municipaux sont sollicités et la phase de négociation préliminaire est alors incontournable. Tous les projets ayant un caractère durable doivent faire l'objet de concertations qui impliquent le directeur de l'établissement, celui-ci devant défendre un projet de collectivité devant des représentants de la Ville. Or, cela suppose l'appropriation par toute l'équipe pédagogique d'un projet qui émane souvent d'un seul enseignant engagé initialement. Lorsque l'action n'est pas inscrite au préalable dans le projet d'école, cela n'est pas sans engendrer quelques différends. D’emblée, la question de la pérennisation et du suivi pédagogique des aménagements demandés est posée par la Ville. Lorsque le projet est soutenu par un enseignant isolé et qu'il est donc le fruit d'une initiative individuelle, et non pas d'une concertation collective, sa durée de vie risque de se limiter à la durée en poste de la personne dans l'école. Par conséquent, moins une action revêt un caractère définitif, plus elle sera facilement acceptée par les institutions concernées. Ainsi, des compromis ont été trouvés avec les jardins pédagogiques. La Ville propose des grands bacs garnis de terre, faciles à déplacer au besoin et n'obligeant pas à casser le revêtement artificiel de la cour. Aussi, inscrire des actions de revitalisation pérennes dans ce contexte où le non-durable est un choix confortable commande des convictions solides et partagées.

\section{Des efforts récompensés}

En dépit des éventuelles difficultés apparaissant à différents niveaux (enseignant, équipe pédagogique, établissement, Ville, institution), les projets de cour qui ont abouti en mobilisant une partie d'école ou toute une école se sont soldés par un sentiment partagé de grande satisfaction. Les plantations de haies champêtres ont suscité beaucoup d'enthousiasme, aussi bien chez les adultes que chez les élèves acteurs du projet. Les habitants des quartiers ont apparemment bien accepté ces 
bandes de verdure composées d'espèces rustiques. Les élèves continuent d'entretenir leur haie (ramassage des papiers, arrosage, paillage...) avec le sentiment d'en être collectivement responsables. Les opérations de plantation ont été médiatisées afin de valoriser le travail des enfants et de garantir la pérennisation de l'action qui demande des soins à programmer dans le temps. Dans tous les cas, les élèves ont dû penser, imaginer leur haie et ils ont dû acquérir de nombreuses compétences tant spécifiques que transdisciplinaires. L'avènement du printemps leur apporte désormais une joie que seule la nature peut procurer aux enfants : les arbres font leurs nouvelles feuilles et certains se couvrent de fleurs à pétales colorés. Et lorsque des enfants ont mis eux-mêmes en terre des petits plants, comme pour une seconde naissance, sur leur espace de jeu, ils savent les voir pousser sans que l'adulte intervienne.

(Re)devenir heureux. C'est le sentiment maintes fois exprimé par des élèves avec qui j'ai partagé ces projets aboutis. Je pourrais relater notre aventure autour du jardin dédié aux papillons, conçu et réalisé dans le patio de l'école, de même que l'enthousiasme pétillant des enfants à l'égard de ces créatures délicates et pour lesquelles ils ont peu à peu développé une certaine empathie en quelques semaines de sensibilisation. Les mots des enfants résument un ensemble de sentiments positifs : «Maintenant, on est heureux quand on voit des papillons dans la rue ou dans le jardin. C'est bien, on peut leur donner des prénoms. » Ils ont vu leur premier papillon dans le patio à la fin de juin et c'était comme un instant de fête.

\section{Quand l'accueil de la nature dans la cour déclenche une démarche globale d'établissement}

Quatre années de partenariat avec une école tourangelle ont permis de mûrir un projet déjà débattu par l'équipe pédagogique à propos de la séparation entre la cour et le boulevard. Cette limite était matérialisée par un grillage posé au-dessus d'un muret de parpaings et doublé par une haie de troènes basse et sévèrement taillée par la Ville. Cette haie monospécifique ne présentait guère d'intérêt sinon celui de permettre aux passants de se débarrasser d'emballages gênants. Les enseignants se plaignaient donc auprès de la mairie de la pollution sonore mais aussi d'un manque de confidentialité. L'équipe pédagogique avait alors opté pour des palissades aveugles afin de garantir ce que les enseignants revendiquaient comme une nécessaire intimité. La Ville n'avait pas répondu favorablement à cette requête et la situation était gelée.

L'idée de planter une haie mixte a alors été soumise en vue d'améliorer le champ visuel de la cour et le paysage très artificialisé du quartier, tout en œuvrant en faveur de la biodiversité locale. Une enseignante avec qui j'ai collaboré en 2003 s'est montrée enthousiaste et sa classe de CM2 a bâti un projet bien argumenté qui n'a pu voir le jour que sur papier. Mais l'idée était bien née. Après plusieurs semaines de classesateliers-environnement, l'exposition à destination des parents et des camarades témoignait de leur motivation. Cependant, l'idée de palissade n'était pas abandonnée et celle de la haie était encore très discutée car sa plantation entraînait une réduction de l'espace de jeu (d'asphalte). L'enseignante porteuse du projet naturaliste ayant été mutée l'année suivante, la cour d'école resta encore deux années scolaires en l'état. 
En 2005, une prise de contact avec le nouveau directeur de l'école fut l'occasion de déterrer le projet de haie. Il fut décidé que la classe de CM2 porterait ce projet, qu'il serait ensuite partagé par toute l'école et surtout qu'il serait intégré au projet d'établissement. Cet engagement collectif a permis que l'enseignante nouvellement affectée s'approprie le projet avec le soutien des collègues et des partenaires, en particulier des classes-ateliers-environnement. Cela a également facilité la transmission du projet d'une année sur l'autre, car sa survie n'est plus liée au bon vouloir d'une personne mais associée au choix politique de l'établissement.

La pérennisation de la haie et de son entretien étant ainsi assurée, l'envie d'inscrire une éducation à l'environnement et au développement durable à l'échelle globale de l'école s'est naturellement imposée. L'effervescence autour de la plantation des petits arbres et le plaisir du travail accompli ensemble ont débouché sur l'idée d'un Agenda 21 d'établissement. La plantation fut l'événement déclencheur d'une démarche communautaire, au-delà d'un simple catalogue d'actions en rapport avec l'environnement. L'école est depuis engagée dans une gouvernance participative où les élèves sont acteurs et représentés lors des réunions du comité de pilotage. La composante nature de l'Agenda 21 apparaît aux enseignants comme essentielle, car l'émergence d'une empathie pour le vivant, l'eau et le sol permet d'envisager de façon responsable et engagée des actions simples pour économiser l'eau ou l'énergie ou encore pour trier les déchets.

Enfin, l'expérience relatée précédemment à l'échelle d'une école de Tours peut nourrir une discussion plus générale à propos de la place accordée à la nature dans une perceptive de développement durable. Cette réflexion est étayée par quelques apports bibliographiques.

\section{La nature de la cour, un terreau fertile pour impulser et entretenir un agenda 21 scolaire}

\section{L'Agenda 21, un projet communautaire}

Les instructions françaises officielles de 2007 relatives à la généralisation de l'éducation au développement durable incitent les établissements scolaires à s'investir dans des démarches vers un développement durable. D’après Cécile FortinDebart et Yves Girault (2005), la gouvernance de ces établissements (procédure qualifiée d'Agenda 21) se tisse autour d'un projet communautaire, donc d'une approche coopérative. Il repose d'abord « sur l'idée que la résolution de la crise environnementale implique davantage un engagement collectif qu'un engagement individuel». Ensuite, il sous-entend la participation des élèves mais aussi des adultes de la communauté scolaire et éducative, ceux-ci devant «tendre à promouvoir deux valeurs qui sont l'exemplarité et la responsabilité». Enfin, le projet suppose des «investigations collectives".

Depuis peu, les engagements se multiplient à travers des projets pédagogiques d'établissement qui embrassent des problématiques globales autant que complexes en s'efforçant d'identifier et de considérer à travers les programmes d'actions communs 
les trois piliers du développement durable communément reconnus. Outre l'incontournable polémique autour de la terminologie utilisée et des valeurs qui soustendent cette volonté affichée de changement, beaucoup s'interrogent sur les entrées idéologiques à privilégier pour engager la communauté et soutenir l'enthousiasme des élèves, surtout chez les plus jeunes pour qui les notions de ressources économiques et de durabilité sont en construction.

\section{Considérer comme bénéfique I'idée de la centralité de l'humain dans la cour de l'école}

À travers cette étude, l'adoption de la posture du scientifique écologue a eu pour effet de stigmatiser l'anthropocentrisme qui s'exerce sur l'écosystème que constitue la cour. Mais dans cet espace tout de même dévolu aux jeux des enfants, cet état de fait n'est pas forcément négatif pour peu que ces jeunes puissent devenir les garants des processus vitaux normaux et compatibles avec les usages anthropiques des lieux. La littérature invite à interroger le cadre philosophique sous-jacent et à donner du sens à nos actions de renaturalisation.

D'abord, il semble que «la crise environnementale est une crise d'usage et de représentations » (Fortin-Debart et Girault, 2005). Aussi, ouvrir le débat investigateur sur une question d'ordre naturaliste concernant les abords immédiats des bâtiments pourrait contribuer à revisiter d'emblée nos conceptions sur la place de l'humain dans le monde et, à notre échelle, dans l'écosphère école. Les recueils de représentations, les débats que la diversité des positionnements va susciter, tout cela devrait converger vers la question de la centralité de l'humain vis-à-vis des êtres vivants, mais aussi des facteurs abiotiques qui conditionnent leur survie : l'eau, le climat, le sol... Dès lors, l'eau ne serait plus appréhendée comme une simple ressource utile à l'Homme, mais comme un patrimoine commun à la communauté vivante dont l'humanité aurait la responsabilité. De même, l'air, au-delà d'une matière inerte essentielle à préserver pour notre santé, pourrait être perçu comme le mélange gazeux idéal dont la stabilisation une fois obtenue a permis la pérennisation du processus vital évolutif entamé il y a quelque trois milliards d'années. Enfin, le sol n'est pas simplement utile à l'agriculture pour nourrir l'humanité, il s'agit d'un monde en soi qui mérite intérêt et émerveillement. Considérer nos paramètres physiques vitaux non plus comme des ressources marchandes mais comme des entités ayant une valeur en soi, car elles incarnent dans leur ensemble l'aventure vitale sur la Terre, serait une introduction à l'éveil de la responsabilité.

L'idée de la centralité de l'humain dans la nature a été notamment abordée par Dominique Bourg (1996). Celui-ci démontre que toute culture est imprégnée par un anthropocentrisme pratique. En effet, les animaux ou les écosystèmes n'ont pas de droits statufiés et la protection dont ils peuvent jouir éventuellement ne repose que sur des considérations d'ordre moral. Dans le travail synthétique de Catherine Larrère (1997), on affirme que la vie sauvage n'est pas morale, qu'elle n'a ni les mêmes règles ni les mêmes valeurs que dans une société d'humains. La souffrance y est nécessaire car fonctionnelle. Ces considérations qui paraissent éloignées de notre cour de récréation constituent pourtant la question centrale dont il faut favoriser la 
dans une démarche d'établissement «vers un développement durable»

La cour avec ses araignées et ses coccinelles peut devenir un lieu suscitant des échanges philosophiques concrets et formateurs avec les enfants. discussion avec les enfants. Leur appréhension de la nature étant profondément anthropomorphique, leur penchant inné à l'empathie peut être contrarié avec l'acquisition de connaissances sur certains comportements animaux: actes de prédation, lutte territoriale... La cour avec ses araignées et ses coccinelles peut devenir un lieu suscitant des échanges philosophiques concrets et formateurs avec les enfants.

Ensuite, Dominique Bourg affirme que l'idée de la centralité de l'humanité serait universelle et que la reconnaissance du caractère externe de cette centralité amène à nous rendre responsables de et pour la nature. Ainsi, le caractère profondément anthropocentrique du concept de développement durable n'a pas forcément une connotation négative, puisque l'on confierait à l'humain la mission d'assurer la continuité du programme vital sur la planète, maintenant que la situation est telle que, sans son intervention positive, toute évolution normale des processus en marche depuis des millénaires est compromise. Le principe de responsabilité développé par Hans Jonas (1990) trouve toute sa substance dans le terrain de jeux des enfants, qu'il soit distillé de façon formelle ou informelle. J'ai souvent été témoin de l'exercice de responsabilité à l'égard du vivant pendant la récréation, du moment que les opérations de revitalisation effectuées dans la cour avaient fait l'objet de débats sur nos droits et devoirs et qu'ils étaient l'aboutissement d'une approche coopérative.

\section{L'épanouissement des éthiques de l'amitié et de la liberté dans la cour d'école}

Un groupe d'enfants à qui l'on aurait confié les soins de la haie, du jardin à papillons, de la zone humide, qui a donc révisé ses idées premières sur la nature par la mise à l'épreuve des débats collectifs, serait plus enclin à considérer les autres problématiques environnementales non directement liées à la biodiversité.

En effet, les enjeux énergétiques liés à l'eau ou aux déchets sont posés par les adultes comme une affaire d'économie des ressources ou même une économie financière. Même si les problèmes ainsi posés sont d'ordre pragmatique, il n'en reste pas moins qu'il s'agit de préoccupations d'adultes et que vouloir motiver les enfants en les projetant dans un avenir incertain relève d'un exercice abstrait, voire voué à l'échec. Les écoliers ne peuvent trouver une motivation efficace pour entreprendre quelque chose dont ils n'auraient qu'une vague conscience des effets, d'autant plus que les situations dans les domaines énergétiques, de distribution d'eau et de traitement des déchets sont toujours extrêmement complexes, même à l'échelle de l'école. Pourquoi apprendre à ne plus gaspiller l'eau, pourquoi éteindre les lumières inutiles? Brandir l'idée inquiétante de manquer un jour d'énergies fossiles ou de manquer d'eau potable ne trouve pas vraiment d'écho évocateur, encore moins enthousiasmant, dans le champ mental de la plupart des enfants.

Aussi, c'est peut-être en (re)suscitant une sensibilité globale, une empathie à l'égard la nature que les enfants deviendraient capables d'établir des échos logiques entre les ressources utiles en eau, en énergie, en matière première et l'état naturel de la mère nourricière. Cette idée rejoint les convictions de l'équipe de recherche de Diane Pruneau dont elle fait état à travers une analyse de projets éducatifs relatifs au 
Des actions en faveur de la nature dans les cours d'école: un levier pertinent pour mieux vivre ensemble

dans une démarche d'établissement «vers un développement durable»

La prise en compte positive de la nature dans l'école contribuerait à l'épanouissement de la compassion et de la générosité envers autrui, d'une part, et alimenterait une force de pensée et un désir d'entreprendre dans la communauté éducative, d'autre part. changement climatique: "Les élèves sont (aussi) passés à l'action parce qu'ils avaient compris que les changements climatiques pourraient avoir des conséquences dans leur milieu et parce qu'ils ont vécu des activités pédagogiques [...] qui leur ont permis de ressentir de l'empathie vis-à-vis l'environnement naturel» (Ouellet et collab., 2005).

Nous rentrons ainsi dans un champ éthique et nous rejoignons les propos de Philippe Zarifian (2008) qui place l'indispensable amour de la nature dans le champ philosophique des éthiques de l'amitié englobant l'amour du prochain dans le sens universel du terme, c'est-à-dire une générosité à l'égard de nos semblables mais étendue à nos compagnons du monde. Philippe Zarifian démontre que la question écologique contemporaine fait évoluer la question éthique, considérant que celle-ci «ne peut pas se limiter aux rapports entre humains et doit obligatoirement englober les rapports à la nature car nous avons le pouvoir de porter atteinte au vivant». Il ajoute que l'éthique de la liberté peut également voler au secours de l'environnement et évoque «la capacité à utiliser les propensions de la nature, à en faire bon usage, ce qui renforce la puissance de la pensée et de l'action de chacun ». Ces compétences apparaissent nécessaires à la bonne gouvernance d'un établissement dynamique qui mobilise les idées novatrices de chacun. Par conséquent, la prise en compte positive de la nature dans l'école contribuerait à l'épanouissement de la compassion et de la générosité envers autrui, d'une part, et alimenterait une force de pensée et un désir d'entreprendre dans la communauté éducative, d'autre part.

Certains sentiments entravent la sensation de liberté nécessaire à un projet collectif. Je constate souvent que les ressentis négatifs entretenus à l'intérieur du groupe classe, souvent engendrés par des jalousies prenant leurs sources dans les multiples détresses de l'humanité, sont des facteurs inhibiteurs puissants. Des disputes, des rancœurs, des sentiments d'injustice agissent négativement et entravent les actions. Inversement, une ambiance sereine et bienveillante agit comme un catalyseur pour le projet. L'exercice régulier de la contemplation de la nature ne pourraitil pas agir comme un baume apaisant pour dépasser ces sentiments négatifs qui nous hantent?

Cette action salvatrice, dans le sens emprunté à Dominique Cottereau (1994), serait favorisée à travers des situations de terrain où l'enfant se passe du médiateur pédagogique faisant habituellement écran ou filtre entre les éléments et les sens des apprenants. L'auteure précitée n'hésite pas à s'immerger dans l'œuvre poétique de Bachelard $(1983,1989)$ qui affirme que la contemplation associée aux exercices sensoriels actifs participe à la rêverie, exercice mental permettant l'établissement de contacts intimes avec la nature. "On ne rêve pas avec des idées enseignées" (Bachelard, 1989). Le philosophe tire de ses épousailles avec les essences vives de la création une sensation de bonheur qu'il explique par un sentiment apaisant d'appartenance au monde. La contemplation ouvre ainsi les portes de la rêverie qui elle-même stimule une imagination attribuant aux éléments naturels comme l'eau, l'air et la terre, un effet moteur sur le monde. Aider les jeunes à comprendre leur place dans leur environnement spatial et temporel, n'est-ce pas accompagner la poursuite de leur (re)mise au monde? 
Ainsi, multiplier les contacts avec la nature ordinaire permettrait aux élèves de butiner ce nectar apaisant et régénérant pour devenir capables de faire ensemble et de considérer de façon responsable et engagée les enjeux environnementaux de ce début de siècle.

\section{Conclusion}

L'intuition initiale selon laquelle les cours d'école, quasi dépourvues de verdure et de la petite faune sauvage normalement attendues, ne sont propices ni au développement des élèves ni à leur bien-être mental est consolidée par la littérature. La réflexion sur le sujet, encore anecdotique en France semble-t-il, mériterait d'être enrichie par une meilleure prise en considération des expérimentations nord-américaines où certains territoires s'engagent plus volontiers sur des projets de revitalisation des lieux de vie des écoles en partenariat avec l'institution éducative. Ainsi l'Association québécoise pour la promotion de l'éducation relative à l'environnement (AQPERE) a-t-elle su imaginer et mener le programme AVEC (Avenir viable École Communauté) qui, d'après Marylène Ferguson (2008), « vise le développement, chez les jeunes, d'une éco-citoyenneté et d'un esprit civique qui s'enracinent dans le lieu privilégié de leur éducation : l'école».

Certes, beaucoup d'actions isolées à vocation naturaliste, essentiellement autour des jardins pédagogiques, reverdissent déjà les lieux consacrés à l'instruction, mais elles s'inscrivent rarement dans un schéma directeur porté par les collectivités et ne répondent pas explicitement à une volonté de transformer nos déserts d'asphalte en espaces de vie suffisamment diversifiés pour aider nos enfants à grandir. Ce constat est transposable à l'échelle de Tours malgré la mise en place d'un dispositif lourd à vocation d'éduquer à l'environnement. L'intransigeance de la réglementation, qui répond à des représentations très ancrées des dangers potentiels liés au sauvage, n'encourage pas les initiatives de verdissement. Mais les difficultés seraient aussi liées au fait que les projets émanent souvent d'individus sans faire d'emblée l'objet d'un projet communautaire. La motivation de l'éducateur et le partenariat instauré ne suffisent souvent pas pour concrétiser des rêves de réintroduction de nature formulés et argumentés par les enfants.

C'est pourquoi les projets de revitalisation des cours, même modestes, parce qu'ils mobilisent souvent différents services municipaux, parce qu'ils font appel aux conseils d'experts, parce qu'ils concernent tous les élèves de l'école qui partagent le même espace, parce qu'ils doivent être expliqués, communiqués et transmis aux cohortes d'élèves à venir, bénéficient de conditions optimales et dynamisantes lorsqu'ils s'intègrent dans une gouvernance participative. Non seulement une telle démarche est une condition de pérennité du projet, mais l'introduction de la nature dans la cour, pourvu que cette idée soit le fruit d'un vrai projet communautaire où les enfants ont une position centrale et que les objectifs pédagogiques soient clairement identifiés et répondent aux exigences du socle commun, peut inversement déclencher et entretenir une démarche de type Agenda 21. L'immersion dans un 
programme éducatif, destiné aussi bien à organiser et réinvestir des savoirs qu'à construire des valeurs, serait d'autant plus efficace que ce programme se nourrirait des choses de la nature ordinaire, elle-même porteuse de messages éducatifs forts.

Ma propre expérience montre l'intérêt de considérer nos cours autrement et d'associer nos enfants à cette aventure. Les enseignants concernés ont été les témoins d'une évolution de leur classe et de l'émergence d'une empathie collective pour le vivant, y compris pour des petites bêtes peu engageantes. Ce nouveau sentiment de compassion à l'égard des arbres, l'herbe, les nuages, l'eau, les araignées... le fait de célébrer la beauté de la création par les jeux sensoriels et l'exercice de compétences plasticiennes préparent les jeunes apprenants à considérer le monde autrement et à s'y engager de façon responsable. L'éducation à l'environnement devient ainsi un levier permettant d'enrichir une démarche d'établissement « vers un développement durable».

\section{Références bibliographiques}

ADAMS, E. (1990). Learning through Landscapes: A Report on The Use, Design, Management and Development of School Grounds. Winchester, Angleterre : Learning through Landscape Trust.

BACHELART, D. (2009). Autobiographie environnementale : explicitation et exploration de l'expérience écoformatrice, dans C. Guillaumin, S. Pesce et N. Denoyel, Pratiques réflexives en formation, ingéniosité et ingénieries émergentes, Paris: L'Harmattan, 125-154.

BACHELARD, G. (1983). Le droit de rêver. Paris: Presses universitaires de France.

BACHELARD, G. (1989). La poétique de la rêverie. Paris : Presses universitaires de France.

BACHELART, D. (2009). Autobiographie environnementale : explicitation de l'expérience écoformatrice et démarche réflexive, dans C. Guillaumin, Ingénierie de la réflexivité. Paris: L'Harmattan.

BOURG, D. (1996). L'Homme artifice. Coll. Le débat. Paris : Gallimard.

CHESKEY, E. et PEARCE, C. (1994). Habitat restoration: changing the schoolyard changes behaviour. FWTAO Newsletter, septembre-octobre.

COBB, E. (1977). The Ecology of Imagination in Childhood. New York: Columbia University Press.

COTTEREAU, D. (1994). À l'école des éléments. Écoformation et classe de mer, Lyon: Chronique sociale. 
FERGUSON, M. (2008). Le programme AVEC, une formation au développement durable et à l'éco-citoyenneté. Communication présentée au Colloque Éducation au développement durable: de l'école au campus, Université d'Albi, juin.

FORTIN-DEBART, C. et GIRAULT, Y. (2005). Agenda 21 : une approche coopérative. POUR, La revue du groupe de recherche pour l'éducation et la prospective, 187, 124-130.

JONAS, H. (1990). Le principe responsabilité. Une éthique pour la civilisation technologique. Paris : Éd. du Cerf.

LARRÈRE, C. (1997). Les philosophies de l'environnement. Paris : Presses universitaires de France.

LIEBERMAN, G. et HOODY, L. (1998). Closing the Achievement Gap: Using the Environment as An Integrating Context for Learning. San Diego : State Education and Environment Roundtable.

MOORE, R. (1989). Before and after asphalte: diversity as an ecological measure of quality in children's outdoor environnement, dans M.N. Block et A.D. Pellegrini (éd.), The Ecological Context of Children's Play. Norwood, NJ : Ablex.

OUELLET, E., LANGIS J., PRUNEAU, D. et GÉLINAS, V. (2005). Comment encourager des comportements responsables à l'égard de l'environnement? Université de Moncton. [En ligne].

http://www.elements.nb.ca/theme:edu_2005/diane/pruneau.htm. Consulté en juillet 2008.

PRUNEAU, D. et DESJARDINS, G. (2003). L'éducation au développement urbain : quelle éducation pour quels développements? Éducation relative à l'environnement, 4, 97-111.

PRUNEAU, D., GRAVEL, H. et OUATTARA, I. (2002). Les relations que les adolescents entretiennent avec leur environnement. Revue des sciences de l'éducation, 28(3), 565-586.

RAFFAN, J. (2002). La nature nourricière: une étude du potentiel d'apprentissage dans les cours d'école. Coll. Le coffre à outils d'Evergreen, Evergreen et Toyota Canada inc.

TITMAN, W. (1994). Special Places; Special People: The Hidden Curriculum of School Grounds. Surrey, Angleterre : Fonds mondial pour la nature.

ZARIFIAN, P. (2008). Morale et éthique face à la question écologique. Communication présentée au Congrès du Réseau École et Nature. Entre pratique et éthique... Des praticiens d'éducation à l'environnement en recherche de cohérence avec leurs valeurs. Cherbourg, mars. 